\title{
Lessons learned from the first outdoor test of perovskite/silicon tandem solar cells
}

\author{
Erkan Aydin*a, Thomas G. Allen ${ }^{\mathrm{a}}$, Michele De Bastiani ${ }^{\mathrm{a}}$, Lujia Xu ${ }^{\mathrm{a}}$, Esma Ugur ${ }^{\mathrm{a}}$, Ahmed Ali \\ Ahmed $^{\mathrm{a}}$, Michael Salvador ${ }^{\mathrm{a}}$, Emmanuel Van Kerschaver $^{\mathrm{a}}$, Stefaan De Wolf ${ }^{\mathrm{a}}$ \\ ${ }^{a}$ King Abdullah University of Science and Technology (KAUST), KAUST Solar Center (KSC), \\ Physical Sciences and Engineering Division (PSE), Thuwal 23955-6900, Kingdom of Saudi Arabia,
}

\begin{abstract}
Perovskite/silicon tandem solar cells promise power conversion efficiencies (PCE) beyond the thermodynamic limit of single-junction devices. This potential has been unveiled via several champion devices, however, their actual outdoor performance is yet to be investigated. Here, we fabricate $25 \%$-efficient two-terminal (2T) monolithic perovskite/silicon tandem solar cells and test them outdoors to reveal the characteristics of these devices specifically in hot and sunny climates, which are the ideal locations to operate such efficient photovoltaic devices. In this article, we summarize our observation on the perovskite/silicon tandem solar cells under actual operational conditions and discuss the lessons we take from our interpretations.
\end{abstract}

Keywords: perovskites, silicon, tandem solar cells, textured tandems, outdoor test, temperature-dependent performance, bandgap

\section{INTRODUCTION}

The field of perovskite/silicon tandem solar cells is a rapidly evolving research area since these tandem cells promise to achieve PCEs beyond the physical limits of their individual single-junction sub cells. The facile perovskite fabrication process, and its versatility in terms of bandgap engineering (the perovskite bandgap can be easily 'tuned' by manipulating its composition) means that an optimal tandem configuration can be achieved in an economically feasible way compared to other approaches that use expensive III/V materials. Indeed, as well as several research groups, already a number of companies have focused on the development of perovskite/silicon tandems.[1-6] However, the actual outdoor performance of such solar cells was yet to be studied and understood. Critically, outdoor tests are a crucial step to understand the real performance of the solar cells before the market entrance.

Coupling silicon solar cells with the with the ideal band gap $\left(E_{\mathrm{g}} \sim 1.73 \mathrm{eV}\right)$ top cell (either perovskite or any other absorber materials) pushes the radiative-efficiency limit of such devices to $\sim 44 \%$ under standard test conditions (STC). However, for monolithic, 2T tandems, this requires perfect current matching between perovskite top cell and c-Si bottom cell. For this, the perovskite $E_{\mathrm{g}}$ should be ideal ( 1.73) eV at STC, [7-9] which is significantly larger than those typically used for record single-junction perovskite devices $(\sim 1.50-1.60 \mathrm{eV})$. [10-12] Typically, to widen the $E_{\mathrm{g}}$ of metal halide perovskites, the Br:I halide ratio in the precursor (and thus crystallized film) is increased.[13] However, beyond a Br:I ratio of $\sim 1: 4$ (corresponding to an $E_{\mathrm{g}}$ of $\sim 1.68 \mathrm{eV}$ ), segregated I-rich and $\mathrm{Br}$-rich domains occur upon illumination, thus hindering device performance. $^{[14],[15,16]}$ This issue remains still as an open question although several mitigation strategies have been offered.[16, 17]

Meanwhile, although the solar cells are characterized at STC, the devices may show different behaviors at actual operational conditions, specifically due to the heating effect originated from the parasitic and sub bandgap absorption.[18] Quite generally, the $E_{\mathrm{g}}$ of semiconductors depends on temperature: with increasing temperature, it narrows for c-Si, but interestingly increases for perovskites.[19, 20] This is relevant for photovoltaics (PV), as outdoor field-testing conditions (FTC) often imply elevated operating temperatures of PV modules well above $25^{\circ} \mathrm{C}$, especially for high solar irradiation and space-constrained roof-top applications (due to heat-trapping effects).[21] Therefore, what may be an ideal perovskite $E_{\mathrm{g}}$ for tandems at STC, is likely not an ideal $E_{\mathrm{g}}$ under actual FTC: Firstly, due to the red-shift of the c-Si $E_{\mathrm{g}}$ with temperature, the required perovskite $E_{\mathrm{g}}$ for current matching will already be lower than $1.73 \mathrm{eV}$. Secondly, due to the blueshift of the perovskite $E_{\mathrm{g}}$ with temperature, its optimal $E_{\mathrm{g}}$ measured at STC will be even lower than that at FTC, impacting the design guidelines for efficient tandems. 
In this work, we experimentally explore the impact of temperature-dependent changes of the $E_{\mathrm{g}}$ of perovskites and c-Si on $2 \mathrm{~T}$ tandem performance. To this end, we first fabricated perovskite/silicon tandem devices with certified PCE of $25 \%$, using double-side textured silicon heterojunction (SHJ) bottom cells, coated with perovskite top cells. Later we experimentally measured the tandem devices under actual outdoor conditions using field-test facilities at KAUST. This article discussed the lessons we learned from these outdoor measurements.

\section{RESULTS AND DISCUSSION}

\subsection{Influence of the diurnal and seasonal variations}

We first evaluate the tandem performance under direct sunlight and environmental factors, which is increasingly advocated as this technology progresses towards market readiness.[22] To protect the devices from environmental contaminants such as oxygen and water, the devices were vacuum laminated between glass and a polyolefin (POE) transparent back sheet (see Figure 1a). The solar cells were mounted on a structure with a tilt angle of $25^{\circ}$ and South orientation. The structure is located in the outdoor testing field at the KAUST campus, near the village of Thuwal, Saudi Arabia (coordinates 22.302494, 39.110737) as shown in Figure 1b. We acquired the spectral data of the test location for one of the test day and we note that the solar spectrum measured at KAUST is relatively weak in the infrared, compared to the standard AM1.5G spectrum, as shown in Figure 1c. This may affect current matching for tandems optimized for STC, which will be discussed later.[23]

$\mathbf{a}$

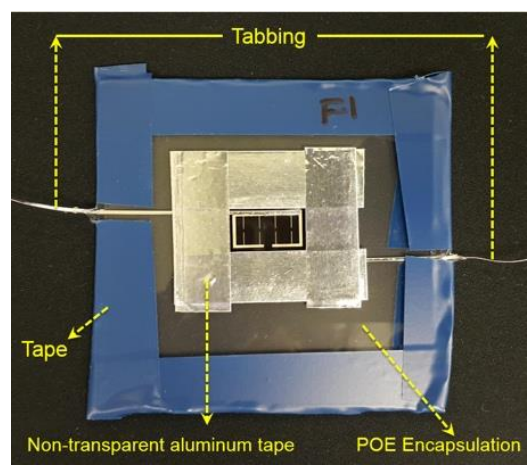

C

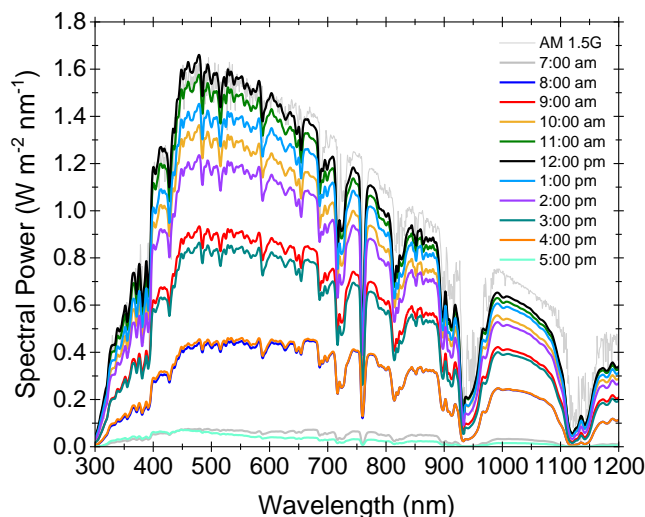

b
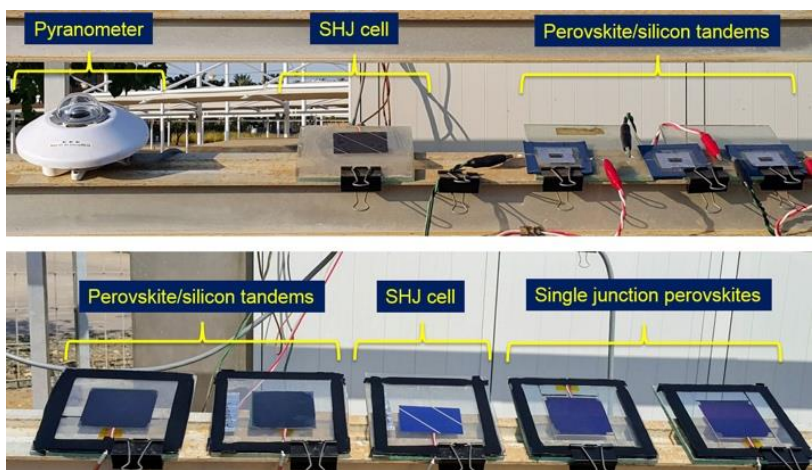

d

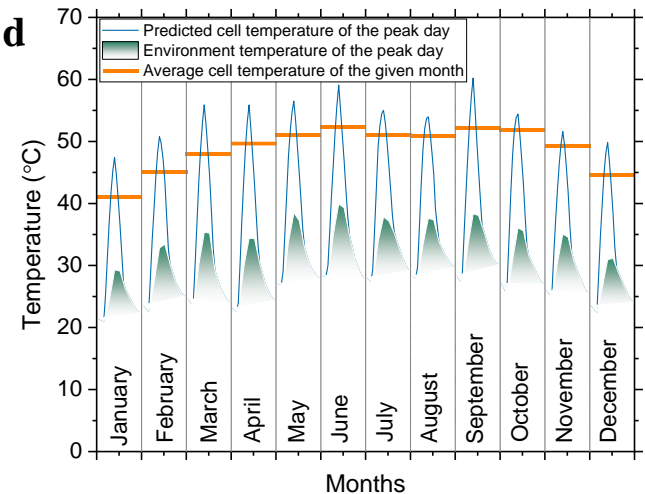

Figure 1. a) The photograph of encapsulated tandem device. b) The photograph of the outdoor testing field at the KAUST. c) Solar spectra over one day measured at KAUST in November 2019. d) Ambient temperature and predicted cell temperature values for perovskite/silicon tandem device over a complete year (based on weather data of 2016, KAUST). The day in each month with the highest peak temperature values are displayed. The orange lines represent the average peak temperature of the month. (The figures are reprinted from [24])

To predict the module temperature for whole year, we first estimated the expected $T_{\text {cell }}$ range under realistic operation conditions. For this, we fabricated $50 \mathrm{~cm}^{2}$ dummy tandems (without front metallization) with a thermocouple attached to their rear, followed by vacuum lamination between two glass sheets with a butyl rubber edge sealant (see Figure 1b). 
These samples were exposed (under open-circuit conditions) to direct sunlight for a week in November 2019. For an ambient temperature $\left(T_{\mathrm{amb}, \max }\right)$ of $38.4^{\circ} \mathrm{C}, T_{\text {cell }}$, max reached up to $60{ }^{\circ} \mathrm{C}$ (Figure 1d). For these outdoor measurements, we used an open-rack test configuration (Figure 1b); significantly higher values for $T_{\text {cell }}$ can be expected in summer, and for roof-top installations because of heat-trap effects.[25] Moreover, the device area here is much smaller than the encapsulation glass. Therefore, we expect that $T_{\text {cell }}$ in commercial-scale modules will be even higher than the reported values here, due to their significantly higher device packing densities. With these data, we then calibrated a devicetemperature model. By combining this model with relevant meteorological data, we simulated the tandem temperature over a complete year (2016), giving a realistic framework for tandem-performance analysis. Based on these calculations, a maximum perovskite/silicon $T_{\text {cell }}$ of $60{ }^{\circ} \mathrm{C}$ was predicted; $\sim 75{ }^{\circ} \mathrm{C}$ appears; therefore, to be realistic for certain rooftop applications and/or under high solar irradiation.[21, 26]

To further investigate the influence of temperature on perovskite/silicon tandems, we calculate the daily energy yield at the radiative limit for the conditions defined by the empirical data of Day 2 (November $7^{\text {th }}, 2019$ ). To do so, we applied the temperature-dependent radiative limit model and integrated, over the course of the day, the energy delivered by the solar cell at the maximum power point for a range of room temperature perovskite bandgaps. To do this, we applied both the measured cell temperature data as the temperature input, and the measured intensity data (Suns) as a factor applied to the AM1.5 spectrum and calculated the optimized efficiency for a range of top cell room temperature bandgaps. The modeled data is presented in Figure 2c below.

Consistent with the modeling presented in the paper, the optimum yield for the $2 \mathrm{~T}$ configuration in Figure 2a occurs at a top cell bandgap of $1.685 \mathrm{eV}$. An interesting metric to note is the relative difference between the maximum energy yield and the energy yield for a top cell $E_{\mathrm{g}}$ of $1.68 \mathrm{eV}$ - that is, the typical upper limit of the perovskite bandgap for which deleterious phase segregation does not occur. When the cell temperature is incorporated into the model, this difference in energy yield is only $0.4 \%$, compared to $3.5 \%$ for the situation where the cell temperature is fixed. Figure $2 \mathbf{b}$ plots the current mismatch between the top and bottom cells in the $2 \mathrm{~T}$ configuration, for the modeling performed in Figure 2a. The data represents the potential top cell contribution to the $J_{\mathrm{sc}}$ minus the possible bottom cell contribution (where, in the $2 \mathrm{~T}$ configuration, the $J_{\mathrm{sc}}$ of the tandem cell is the least of the two values). Above the zero lines, the top cell current dominates, and the $J_{\text {sc }}$ of the tandem device is constrained by the bottom cell. Below zero, the bottom cell dominates the current, and top cell constrains the $J_{\text {sc }}$ of the $2 \mathrm{~T}$ devices.

The effect of temperature on the current mismatch can be seen in the comparison between the solid and dashed lines. The dashed lines, representing the expected behavior of a fixed $T_{\text {cell }}$ of $25^{\circ} \mathrm{C}$, mimic the input intensity curve (grey dotted line) by a linear scaling factor. This scaling factor is 0 when the top and bottom cells are current matched (at $25{ }^{\circ} \mathrm{C}$ ), which occurs for a top cell $E_{\mathrm{g}}$ of $\sim 1.698 \mathrm{eV}$. Since the $T_{\text {cell }}$ in the model does not change, the current matched device remains so throughout the day. The solid lines in the Figure $\mathbf{2 b}$ represent the same scenario when the measured cell temperature is included in the model. The effect of temperature distorts the curves, such that the current mismatch fluctuates throughout the day, slightly broadening the choice of optimal bandgap, as seen in Figure 2a, and thereby impacting the overall daily energy yield.

Performing the same modeling in Figure $\mathbf{2 b}$ but using the measured spectra data of Figure 1c results in a blue shift in the optimal top cell bandgap to $\sim 1.73 \mathrm{eV}$ owing to a relatively higher contribution of blue photons relative to red in the measured spectrum, as shown in the graph below. To mimic the influence of parasitic absorption of glass and encapsulants (e.g., EVA or POE) in a real module, we performed the same modeling but filtered the spectrum of wavelengths up to 350 , 375 and $400 \mathrm{~nm}$ (Figure 2c). Doing so shifts the optimal bandgap back to $\sim 1.70 \mathrm{eV}$ and more than halving the penalty paid in terms of the reduction in the energy yield by limiting the top cell to $1.68 \mathrm{eV}$ from $8 \%$ to $3.8 \%$ for the single day simulated here. A similar process could be applied to the simulations of Figure 2a, which will also redshift the optimal $E_{\mathrm{g}}$ to values below $1.68 \mathrm{eV}$.

We note that, as we have limited spectral data from the test field site where the outdoor experiments were conducted, we cannot comment on the consistency of the blue weighting in the measured spectra throughout the year. Additional data and field tests will be required in future work to further investigate the effect of the spectrum on measured and potential yields of perovskite/silicon tandem cells over longer time spans. Indeed, more realistic cell modeling would be beneficial to calculate energy yields for real devices, that included additional non-ideal losses, including parasitic absorption at the cell and module level, and the interplay between series and shunt resistances on the measured fill factor (FF) in $2 \mathrm{~T}$ tandems 
cells, an effect that tends to mitigate the impact of current mismatch but lowers the overall PCE. We also note that the modeling performed in this paper would benefit from utilizing cell absorption derived not from the temperature-dependent $E_{\mathrm{g}}$ and Urbach energies but from empirically derived temperature and $E_{\mathrm{g}}$ (i.e., composition) dependent absorption data, a general equation for which is currently not available for perovskites. However, the modeling approach presented here illustrates general trends regarding the interplay between temperature, $E_{\mathrm{g}}$, and spectral composition that mitigate the need for further development of wide $E_{\mathrm{g}}$ perovskites in excess of $1.68 \mathrm{eV}$ for applications in perovskite/silicon tandems.
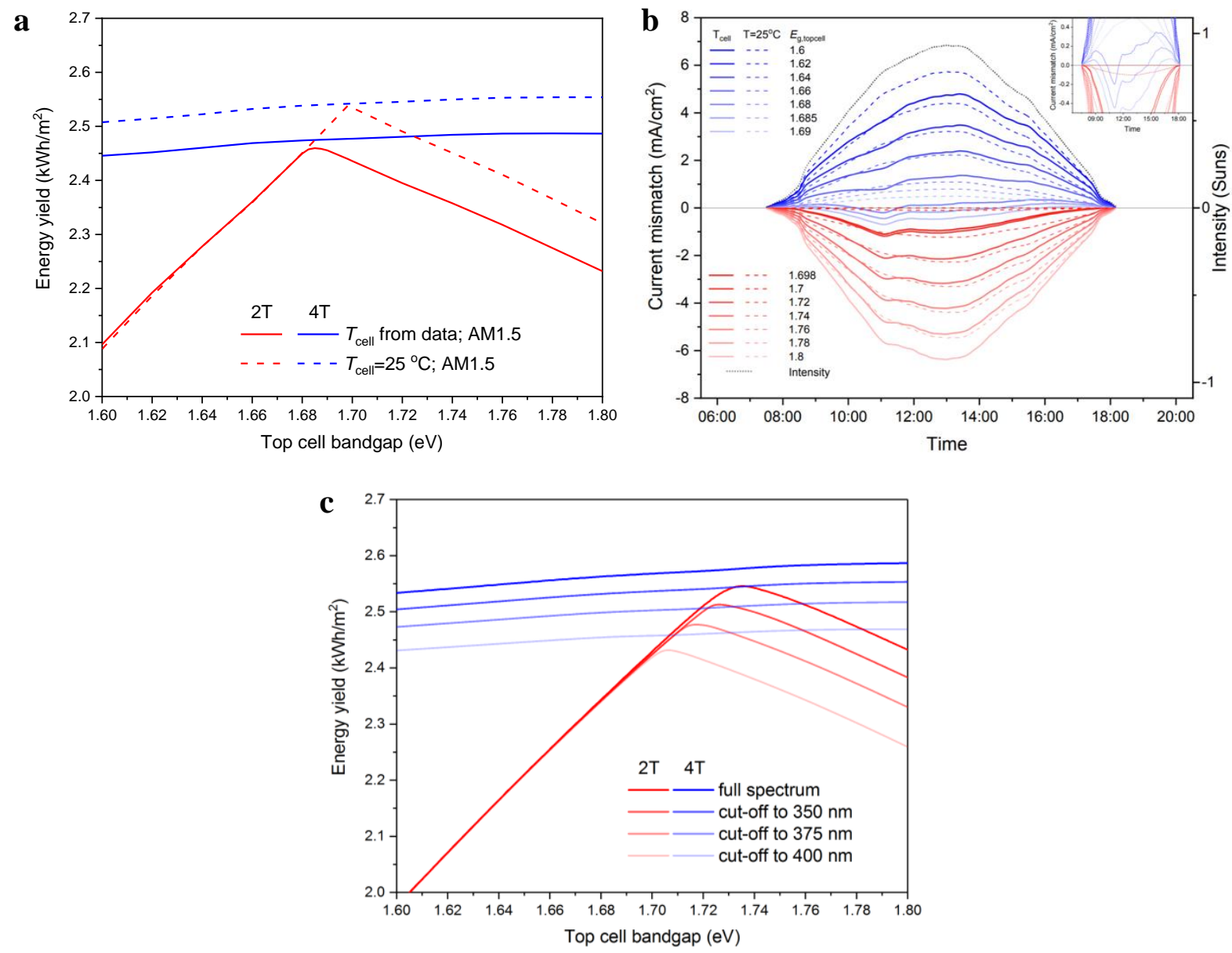

Figure 2. a) Theoretical yield for $2 \mathrm{~T}$ and $4 \mathrm{~T}$ tandems calculated at the radiative limit using data from.[24] Ref. The dashed data represent calculations performed with a fixed cell temperature of $25^{\circ} \mathrm{C}$. b) The current mismatch in the $2 \mathrm{~T}$ calculations is shown as a function of time. c) Theoretical yield for $2 \mathrm{~T}$ and $4 \mathrm{~T}$ tandems calculated at the radiative limit using cell temperature data from November $7^{\text {th }}, 2019$ and spectral data of Figure 1c. To mimic the effects of glass/encapsulant parasitic absorption typical of a module deployed in the field, we have also modeled the yield with the spectrum cut-off for wavelengths below 350,375 , and 400 nm. (The figures are reprinted from [24])

\subsection{Temperature coefficient of the perovskite/silicon tandem solar cells}

We determined the temperature coefficient (TC) of the tandem devices via temperature-dependent $\mathrm{J}-\mathrm{V}$ measurements in a controlled laboratory environment under a simulated AM1.5G spectrum, within the expected operational temperature range, by varying the temperature between 25 and $75^{\circ} \mathrm{C}$ (Figure 3a). Here, we note that TC is defined as the PCE loss per temperature increment, normalized to the PCE at STC. From these data, we calculated the TC for our tandem to be -0.26 $\% \mathrm{~K}^{-1}$ (Figure 3b).[27] The c-Si bottom cell used in this study showed a TC of $-0.23 \% \mathrm{~K}^{-1}$ when normalized at $25^{\circ} \mathrm{C}$; a 
TC of $-0.26 \% \mathrm{~K}^{-1}$ - which is more typical for SHJ technology - was found when normalized at $40{ }^{\circ} \mathrm{C}$.[27] Therefore, the addition of the perovskite top-cell barely affects the tandem TC.

Notably, besides the physical factors that dictate the TC of single-junction solar cells,[28] the TC of 2T tandem solar cells is additionally affected by possible current mismatch between top and bottom cell. In the tandem under study here, we find this increasingly to be the case with temperature, resulting in a decreased tandem $J_{\mathrm{SC}}$, thereby inflating the tandem TC. This current mismatch is due to the $E_{\mathrm{g}}$ shifts of c-Si and perovskite layers with temperature: the c-Si $E_{\mathrm{g}}$ narrows, but the perovskite $E_{\mathrm{g}}$ broadens with temperature. This implies that the tandem TC can be minimized by optimizing the perovskite $E_{\mathrm{g}}$ towards current matching at the highest expected operational temperature. This possible mismatch-induced increase of the tandem TC is also of importance when comparing tandem TCs as well as their PCEs at STC in the literature.
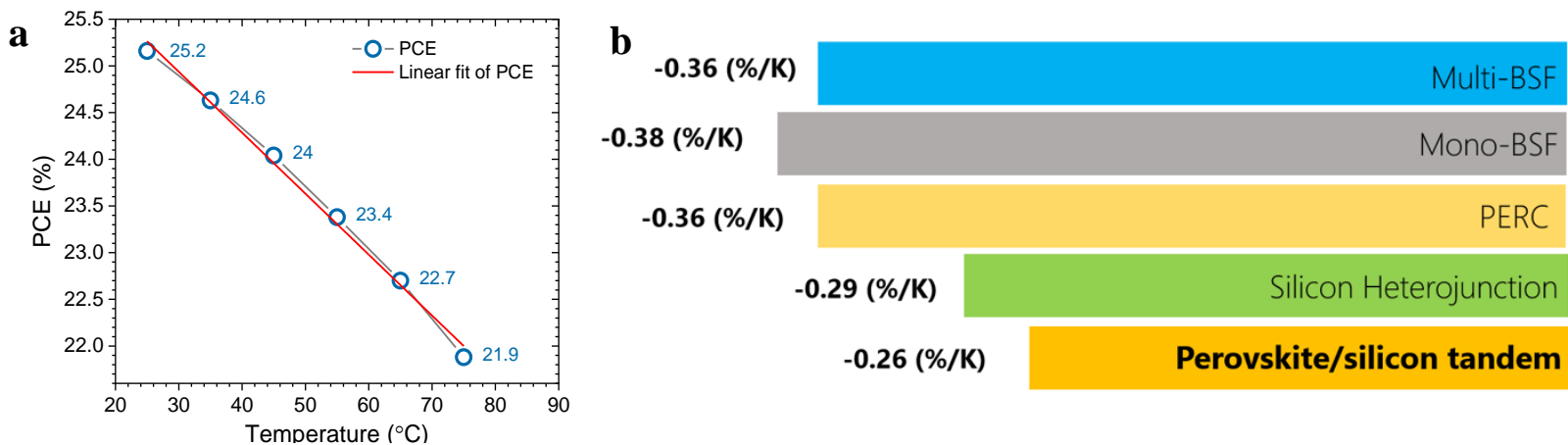

Figure 3. a) The change of the photovoltaic parameters of the perovskite/silicon tandem solar cells by temperature under AM $1.5 \mathrm{G}$ illumination. For the temperature coefficient calculations, the following formula is used and found to be $-0.26 \%{ }^{\circ} \mathrm{K}^{-1}$. Here, the temperature coefficient is normalized at $25{ }^{\circ} \mathrm{C}$ since this value is the standard reference temperature for terrestrial and most space PV technologies. b) The summary of the temperature coefficients of the various c-Si technologies, together with perovskite/silicon tandem solar cells.[18] (The panel a is reprinted from [24]))

To further elucidate the $E_{\mathrm{g}}$ shift as a function of temperature, we studied the temperature-dependent external quantum

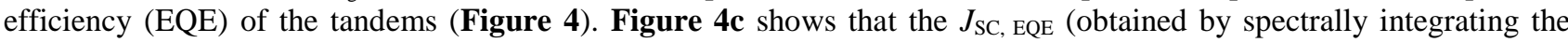
product of EQE and incident solar spectrum) of the c-Si bottom cell increases with temperature. This is due to additional contributions of photons with longer wavelengths (as the c-Si $E_{\mathrm{g}}$ redshifts), as well as photons with shorter wavelengths (as the perovskite $E_{\mathrm{g}}$ blue shifts), coupling more light from two distinct spectral ranges in the c-Si cell underneath. Despite this, the overall tandem $J_{\mathrm{SC}}$ decreases because the $J_{\mathrm{SC}}$, EQE of the perovskite top cell decreases (as its $E_{\mathrm{g}}$ blue shifts), eventually limiting the tandem $J_{\mathrm{SC}}$. Therefore, what may be the optimal perovskite $E_{\mathrm{g}}$ for STC (i.e. at $25{ }^{\circ} \mathrm{C}$ ) may not be so for outdoor applications, where $T_{\text {cell }}$ is often significantly higher.
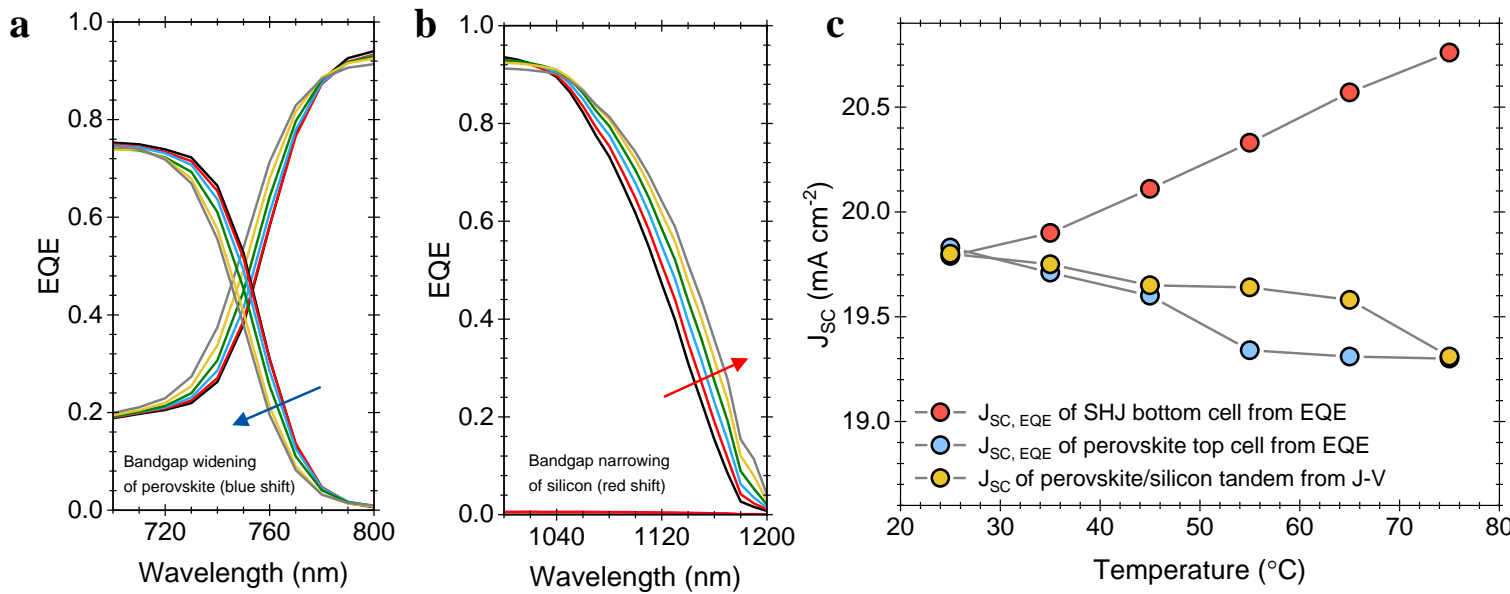
Figure 4. The EQE characteristics of perovskite/silicon tandem devices at a) perovskite, and b) silicon band edges. c) Temperature-dependent change of EQE measured current density (JSC, EQE) values for both sub-cells together with the J-V measured current density ( $\mathrm{JSC}_{\mathrm{SC}}$ ) of a tandem solar cell, showing current limiting conditions. (The figures are reprinted from [24])

\subsection{Temperature correction on the optimal bandgap of the perovskite top cells}

To further elucidate the effect of temperature on current matching in perovskite/silicon 2T tandem solar cells, and provide tandem design guidelines with respect to the perovskite $E_{\mathrm{g}}$, we modeled their temperature-dependent radiative-efficiency limit, based on the modeling methodology outlined by Jean et al.[29] Our adapted model considers the effect of the perovskite $E_{\mathrm{g}}$ and its Urbach energy $\left(E_{U}\right)$ on tandem performance; further details are given in the reference.[24] We have plotted the tandem $J_{\mathrm{SC}}$ as a function of the top cell $E_{\mathrm{g}}$, and include the contributions of the sub-cells to the overall tandem $J_{\mathrm{SC}}$ (dashed and dotted lines in the Figure 5a). For any given temperature, the left side (lower values of perovskite $E_{\mathrm{g}}$ energies) of the peak in current denotes the values of the perovskite $E_{\mathrm{g}}$ that induce a constraint in the overall tandem $J_{\mathrm{SC}}$ by the c-Si bottom cell. Here, the c-Si subcell is shaded by the top cell and so the tandem $J_{\mathrm{SC}}$ follows the Si sub-cell current and its temperature dependence. On the right side of the apex, the wide- $E_{\mathrm{g}}$ perovskite current is constraining the device.

The inversion in the trend of temperature at high perovskite $E_{\mathrm{g}}$ is due to the asymmetry in the trend of $E_{\mathrm{g}}$ with temperature, as also seen in the EQE(T) spectra of Figure 4a and $\mathbf{4 b}$. When the tandem is current limited by the bottom cell, the increase in temperature induces an increase in infrared absorption and so an increase in the tandem $J_{\mathrm{SC}}$. The opposite is true when the tandem is constrained by the top cell: increasing the temperature results in lower currents in the top cell due to further broadening of the $E_{\mathrm{g}}$ away from the current matching condition (Figure 4c), as also demonstrated experimentally. By adding the effect of band tailing to the radiative-efficiency limit calculations, we show that the ideal perovskite $E_{\mathrm{g}}$ value decreases substantially from the traditional calculation; this value decreases further with increasing temperature (Figure 5b). Critically, the optimal perovskite $E_{\mathrm{g}}$ value at the radiative limit decreases to below $1.68 \mathrm{eV}$ for relevant device operating temperatures; such lower bandgaps are typically associated with materials with modest Br:I ratios, that are therefore more stable.
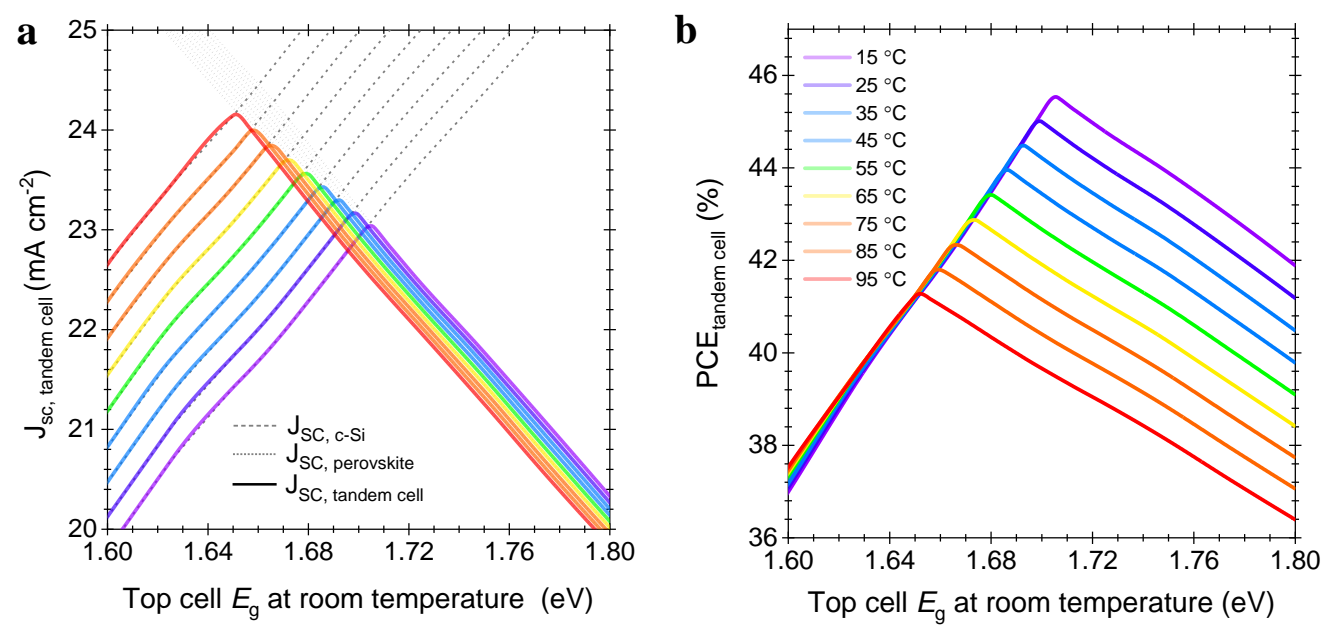

Figure 5. a) The variation of the total $2 \mathrm{~T}$ tandem cell current as a function of the top cell perovskite $E_{\mathrm{g}}$ and cell temperature. The dotted lines show the current contribution from the perovskite subcell, while the dashed lines show the contribution from the c-Si subcell. b) Calculated PCE values for both $2 \mathrm{~T}$ (solid lines) and 4T (dotted lines) configurations as a function of top cell $E_{\mathrm{g}}$ and temperature. (The figures are reprinted from [24]) 


\section{CONCLUSIONS}

We fabricated fully-textured monolithic perovskite/silicon tandem solar cells with a certified efficiency of $25 \%$ and the highest $J_{\mathrm{SC}}$ value report to date for such devices. We then studied the interplay between the operating temperature and the bandgaps of the sub-cells, and its effect on tandem performance with specific attention to elevated temperatures typical of device operating conditions. We tested these devices under controlled lab and under outdoor field conditions. We take several lessons from these measurements and characterizations.

(i) We find that perovskite/silicon tandem cells can easily reach $\sim 60{ }^{\circ} \mathrm{C}$ under direct sunlight and calculated that this can be as high as $\sim 75^{\circ} \mathrm{C}$ over the course of a year during operation. These observations set a framework for the optimal design of silicon/perovskite based tandem cells towards maximized power output under actual operating conditions.

(ii) $\mathrm{TC}$ of the perovskite/silicon tandems $\left(-0.26 \% \mathrm{~K}^{-1}\right)$ is close to the SHJ bottom cells, and much better than other market dominating silicon technologies. The TC of the perovskite/silicon tandem can be decreased further, which implies less temperature sensitive PV modules can be produced.

(iii) With increasing temperature, the $E_{\mathrm{g}}$ of c-Si narrows while for perovskites it broadens, which affects currentmatching conditions. Therefore, for optimal tandem performance at field-testing conditions, we find that the perovskite $E_{\mathrm{g}}$ at $\mathrm{STC}\left(25^{\circ} \mathrm{C}\right)$ should be significantly lower compared to earlier findings. These findings were further supported by calculations using a modified radiative-efficiency limit model. In doing so, we find that the optimal perovskite $E_{\mathrm{g}}$ falls below $1.68 \mathrm{eV}$ (measured at $25^{\circ} \mathrm{C}$ ). This implies that conventional perovskites with moderate Br:I ratios can be used for tandems, which do not suffer from halide segregation.

(iv) While selecting the ideal perovskite top cells bandgap, the climatic conditions of the operation locations should be considered. This implies the necessity of more the development of the more customized tandem module manufacturing.

(v) More outdoor tests should be performed with longer measurements periods to fully figure out the long-term operational issues of the perovskite/silicon tandem solar cells. Although we used industry standard encapsulation methodologies, the fast degradation of the devices in the field reveals the necessity of developing alternative encapsulation techniques.

\section{ACKNOWLEDGEMENTS}

The research reported in this publication was supported by funding from King Abdullah University of Science and Technology (KAUST) under award nos. OSR-CARF URF/1/3079-33-01 and IED OSR-2019-4208. The authors thank TUV Rheinland Group for providing solar spectra from their outdoor test field on the KAUST campus.

\section{REFERENCES}

[1] Y. Hou, E. Aydin, M. De Bastiani et al., "Efficient tandem solar cells with solution-processed perovskite on textured crystalline silicon," Science, 367(6482), 1135-1140 (2020).

[2] A. Al-Ashouri, E. Köhnen, B. Li et al., "Monolithic perovskite/silicon tandem solar cell with \&gt;29\% efficiency by enhanced hole extraction," Science, 370(6522), 1300-1309 (2020).

[3] J. Xu, C. C. Boyd, Z. J. Yu et al., "Triple-halide wide-band gap perovskites with suppressed phase segregation for efficient tandems," Science, 367(6482), 1097-1104 (2020).

[4] F. Sahli, J. Werner, B. A. Kamino et al., "Fully textured monolithic perovskite/silicon tandem solar cells with $25.2 \%$ power conversion efficiency," Nature Materials, (2018). 
[5] H. Shen, S. T. Omelchenko, D. A. Jacobs et al., "In situ recombination junction between p-Si and $\mathrm{TiO}<\mathrm{sub}>2</$ sub $>$ enables high-efficiency monolithic perovskite/Si tandem cells," Science Advances, 4(12), eaau9711 (2018).

[6] E. Aydin, J. Liu, E. Ugur et al., "Ligand-bridged charge extraction and enhanced quantum efficiency enable efficient n-i-p perovskite/silicon tandem solar cells," Energy \& Environmental Science, (2021).

[7] G. E. Eperon, M. T. Hörantner, and H. J. Snaith, "Metal halide perovskite tandem and multiple-junction photovoltaics," Nature Reviews Chemistry, 1, 0095 (2017).

[8] T. Leijtens, K. A. Bush, R. Prasanna et al., "Opportunities and challenges for tandem solar cells using metal halide perovskite semiconductors," Nature Energy, (2018).

[9] S. Albrecht, and B. Rech, "Perovskite solar cells: On top of commercial photovoltaics," Nature Energy, (2017).

[10] Q. Jiang, Y. Zhao, X. Zhang et al., "Surface passivation of perovskite film for efficient solar cells," Nature Photonics, (2019).

[11] J. J. Yoo, S. Wieghold, M. C. Sponseller et al., "An interface stabilized perovskite solar cell with high stabilized efficiency and low voltage loss," Energy \& Environmental Science, 12(7), 2192-2199 (2019).

[12] X. Zheng, Y. Hou, C. Bao et al., "Managing grains and interfaces via ligand anchoring enables 22.3\%efficiency inverted perovskite solar cells," Nature Energy, (2020).

[13] K. A. Bush, K. Frohna, R. Prasanna et al., "Compositional Engineering for Efficient Wide Band Gap Perovskites with Improved Stability to Photoinduced Phase Segregation,” ACS Energy Letters, 428-435 (2018).

[14] S. Mahesh, J. M. Ball, R. D. J. Oliver et al., "Revealing the Origin of Voltage Loss in Mixed-Halide Perovskite Solar Cells," Energy \& Environmental Science, (2019).

[15] J.-P. Correa-Baena, M. Saliba, T. Buonassisi et al., "Promises and challenges of perovskite solar cells," Science, 358(6364), 739-744 (2017).

[16] E. Aydin, M. De Bastiani, and S. De Wolf, "Defect and Contact Passivation for Perovskite Solar Cells," Advanced Materials, 0(0), 1900428 (2019).

[17] F. H. Isikgor, F. Furlan, J. Liu et al., "Concurrent cationic and anionic perovskite defect passivation enables $27.4 \%$ perovskite/silicon tandems with suppression of halide segregation," Joule, 5(6), 1566-1586 (2021).

[18] L. Xu, W. Liu, H. Liu et al., "Heat generation and mitigation in silicon solar cells and modules," Joule, 5(3), 631-645 (2021).

[19] M. Ledinsky, T. Schönfeldová, J. Holovsky et al., "Temperature Dependence of the Urbach Energy in Lead Iodide Perovskites,” The Journal of Physical Chemistry Letters, (2019).

[20] P. Singh, and N. M. Ravindra, "Temperature dependence of solar cell performance — an analysis," Solar Energy Materials and Solar Cells, 101, 36-45 (2012).

[21] T. Sample, and A. Virtuani, "Modification to the standard reference environment (SRE) for nominal operating cell temperature (NOCT) to account for building integration," Proc. of the 24th Eu-PVSEC, Hamburg, 3332 (2009).

[22] M. V. Khenkin, E. A. Katz, A. Abate et al., "Consensus statement for stability assessment and reporting for perovskite photovoltaics based on ISOS procedures," Nature Energy, 5(1), 35-49 (2020).

[23] M. T. Horantner, and H. Snaith, "Predicting and Optimising the Energy Yield of Perovskite-on-Silicon Tandem Solar Cells under Real World Conditions," Energy \& Environmental Science, (2017).

[24] E. Aydin, T. G. Allen, M. De Bastiani et al., "Interplay between temperature and bandgap energies on the outdoor performance of perovskite/silicon tandem solar cells," Nature Energy, (2020).

[25] J. Kurnik, M. Jankovec, K. Brecl et al., "Outdoor testing of PV module temperature and performance under different mounting and operational conditions," Solar Energy Materials and Solar Cells, 95(1), 373-376 (2011).

[26] T. Nordmann, and L. Clavadetscher, "Understanding temperature effects on PV system performance." 3, 22432246 Vol.3.

[27] J. Haschke, J. P. Seif, Y. Riesen et al., "The impact of silicon solar cell architecture and cell interconnection on energy yield in hot \& sunny climates," Energy \& Environmental Science, 10(5), 1196-1206 (2017).

[28] O. Dupré, R. Vaillon, and M. A. Green, "Physics of the temperature coefficients of solar cells," Solar Energy Materials and Solar Cells, 140, 92-100 (2015).

[29] J. Jean, T. S. Mahony, D. Bozyigit et al., "Radiative Efficiency Limit with Band Tailing Exceeds 30\% for Quantum Dot Solar Cells,” ACS Energy Letters, 2(11), 2616-2624 (2017). 